\title{
THE HERBIG BE STAR V1818 ORI AND ITS ENVIRONMENT
}

\author{
Hsin-Fang Chiang ${ }^{1}$, Bo Reipurth ${ }^{1}$, and Lynne Hillennbrand ${ }^{2}$ \\ ${ }^{1}$ Institute for Astronomy and NASA Astrobiology Institute, University of Hawaii at Manoa, 640 North Aohoku Place, \\ Hilo, HI 96720, USA; hchiang@ifa.hawaii.edu, reipurth@ifa.hawaii.edu \\ 2 Department of Astronomy, California Institute of Technology, Pasadena, CA 91125, USA \\ Received 2014 May 8; accepted 2015 January 9; published 2015 February 26
}

\begin{abstract}
The little-studied Herbig Be star V1818 Ori is located in the direction of the southern L1641 cloud and the Mon R2 star-forming complex, and is most likely associated with the latter at a distance of $\sim 900$ pc. A high-resolution spectrum is consistent with a spectral type around $\mathrm{B} 7 \mathrm{~V}$, with lines of $\mathrm{H} \alpha$, the red $\mathrm{Ca}$ II triplet, and several forbidden lines in emission. An All Sky Automated Survey V-band light curve spanning 9 yr reveals major variability with deep absorption episodes reminiscent of the UX Orionis stars. We have searched for additional young stars clustering around V1818 Ori using grism images and the 2MASS and Wide-field Infrared Survey Explorer catalogs, and have found almost two dozen fainter stars with evidence of youth. Direct images show that the bright star IRAS 05510-1025, only about 3 arcmin from V1818 Ori, is surrounded by a reflection nebula, indicating its association with a molecular cloud. A spectrum of the star shows no emission-lines, and it is found to be a close binary with late $\mathrm{B}$ and early $\mathrm{G}$ type components. Its radial velocity indicates that it is an interloper, accidentally passing through the cloud and not physically associated with V1818 Ori.
\end{abstract}

Key words: stars: individual (V1818 Ori) - stars: emission-line, Be - stars: variables: T Tauri, Herbig Ae/Be

\section{INTRODUCTION}

Herbig Ae/Be stars, first identified by Herbig (1960), are pre-main-sequence stars of intermediate mass and the more massive counterparts of $\mathrm{T}$ Tauri stars. They are visible at optical wavelengths, often associated with nebulosity, and show infrared excess from circumstellar disks. A large number of Herbig Ae/Be stars have been identified by now (e.g., Thé et al. 1994; Malfait et al. 1998; Vieira et al. 2003). These intermediate-mass stars bridge several transitions among young stars such as the formation regimes of solar-type stars and massive stars, between slow and fast rotators, and between stars with convective and radiative interior structures. Given the shape of the initial mass function, it is to be expected that these intermediate stars should be accompanied with small clusters of low-mass stars, and such clusterings have been discovered around some Herbig Ae/Be stars (Hillenbrand 1995; Testi et al. 1998, 1999), although much remains to be done in this area. Modern instruments with high spatial and spectral resolution have taken advantage of the brightness of Herbig $\mathrm{Ae} / \mathrm{Be}$ stars at both optical and infrared wavelengths to obtain detailed information on selected stars, as summarized in the recent ESO Workshop on these stars. ${ }^{3}$ The discovery and documentation of new bona fide members of the class of Herbig Ae/Be stars thus allows a wide range of more detailed observations.

V1818 Ori (also known as IRAS 05513-1024) is a Herbig $\mathrm{Ae} / \mathrm{Be}$ star $(V \sim 13)$ located toward the southern end of the L1641 cloud in Orion. Attention was first drawn to this star by Gregorio-Hetem et al. (1992), who suspected it might be a Herbig Ae/Be star, which was later confirmed spectroscopically by Vieira et al. (2003). At large scale, Lee \& Chen (2009) showed that V1818 Ori is associated with the GN 05.51.4 cloud near NGC 2149 along the edge of the Orion-Eridanus Superbubble. At small scale, it is associated with a bright arcshaped reflection nebula to the northeast (Magakian 2003; see

See http://www.eso.org/sci/meetings/2014/haebe2014/program.html
Figure 1). The $L^{\prime}$ band image by Connelley et al. (2008) resolved V1818 Ori into a visual binary with a separation of about 3."5. V1818 Ori was included in the near-infrared survey of Class I protostars by Connelley \& Greene $(2010,2014)$ and strong veiling was found.

We have been puzzled by the presence of this Herbig $\mathrm{Ae} / \mathrm{Be}$ star toward the southern end of the L1641 cloud, which is rather inactive in star formation. We have also been interested in the presence of another bright nebulous star, IRAS 05510-1025 about $\sim 3.2$ west of V1818 Ori, indicating the possibility that V1818 Ori could be a rare example of a wide pair of Herbig $\mathrm{Ae} / \mathrm{Be}$ stars. In this paper we examine these aspects and present new data from optical spectroscopy and infrared imaging of V1818 Ori and its surroundings. Section 2 describes the observations and data reduction. Section 3 discusses the results from the new data as well as archival data, with the conclusions in Section 4.

\section{OBSERVATIONS AND DATA REDUCTION}

\subsection{Near-infrared Imaging and Photometry}

Near-infrared observations of the V1818 Ori field were carried out on UT 2012 Jan 10 with the Subaru telescope using the Infrared Camera and Spectrograph (IRCS; Tokunaga et al. 1998; Kobayashi et al. 2000). The images were taken with the $J(1.25 \mu \mathrm{m}), H(1.64 \mu \mathrm{m})$, and $K^{\prime}(2.15 \mu \mathrm{m})$ filters of the Mauna Kea Observatories (MKO) system (Simons \& Tokunaga 2002; Tokunaga et al. 2002). The weather conditions were photometric with a seeing of $\sim 0.9$ at the $K^{\prime}$ band. The image scale is 52 mas per pixel and the field of view is $54^{\prime \prime}$ on a side. For each filter, a five-point dithering was used with a step size of $4^{\prime \prime}$ to map the region around and north of V1818 Ori. The airmass during the observations was around 1.2.

The data reduction was done using the Image Reduction and Analysis Facility (IRAF; Tody \& Crawford 1986), following the procedures described in Connelley et al. (2008) and the 


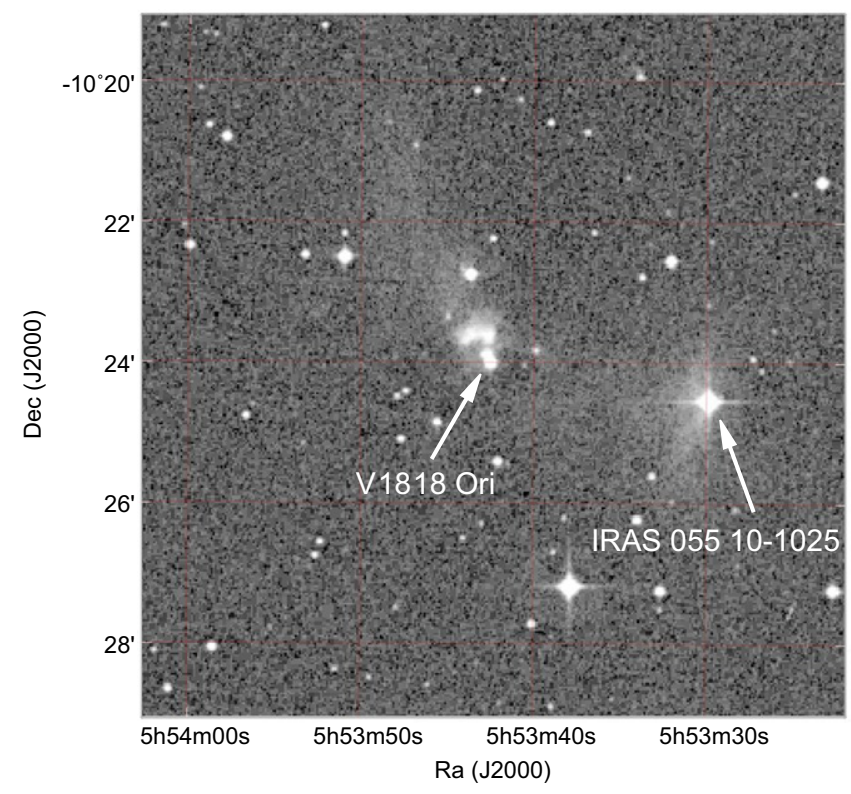

Figure 1. $B_{j}$-band image of the V1818 Ori vicinity from the Digitized Sky Survey (DSS) taken by the UK Schmidt telescope. V1818 Ori (IRAS 05513-1024) is located at the center of the field, surrounded by structured and clumpy reflection nebulosity. The star IRAS $05510-1025$ to the west of V1818 Ori is also associated with a (more diffuse) reflection nebulosity.

IRCS cookbook. ${ }^{4}$ The frames were dark subtracted, flattened using sky flats, and sky subtracted. Objects were masked before combining for deriving flat and sky frames. Then the frames were aligned and averaged for photometric measurements. A three-color composite image of $J, H$, and $K^{\prime}$ bands is shown in Figure 2.

Following Connelley et al. (2008), aperture photometry was performed using IMEXAMINE in IRAF with five aperture sizes $(0.9,1.2,1.5,1 . ! 8$, and 2.1$)$. The standard stars were measured in the same way and the magnitudes from the UKIRT Faint Standards (Leggett et al. 2006) were adopted to give magnitude estimates of the targets. The $K$ magnitude from the standard catalog is used as the standard $K^{\prime}$ magnitude. This is an acceptable approximation because the $K$ and $K^{\prime}$ filters have similar bandwidth in the Mauna Kea Observatories Nearinfrared filter set. Also, the standard stars used are early A type stars which further mitigate the difference. Measurements using different aperture sizes and different standard stars are averaged and errors are estimated. Furthermore, a minimum photometric error is estimated by the standard deviation of the standard star photometry. For each target, the error is determined by the standard deviation of individual measurements using different standard stars and different aperture sizes. The two errors are combined by a Pythagorean sum to represent the overall photometric uncertainty.

For airmass correction, we use the median extinction values from Krisciunas et al. (1987). The extinction coefficient value for the $K$ band is used for our $K^{\prime}$ observations. The error introduced by this is of the order of $0.001 \mathrm{mag}$ in terms of airmass correction, and much smaller than the quoted errors. A list of identified sources in the observed field, the photometric results, and the estimated errors are given in Table 1.

http://subarutelescope.org/Observing/DataReduction/

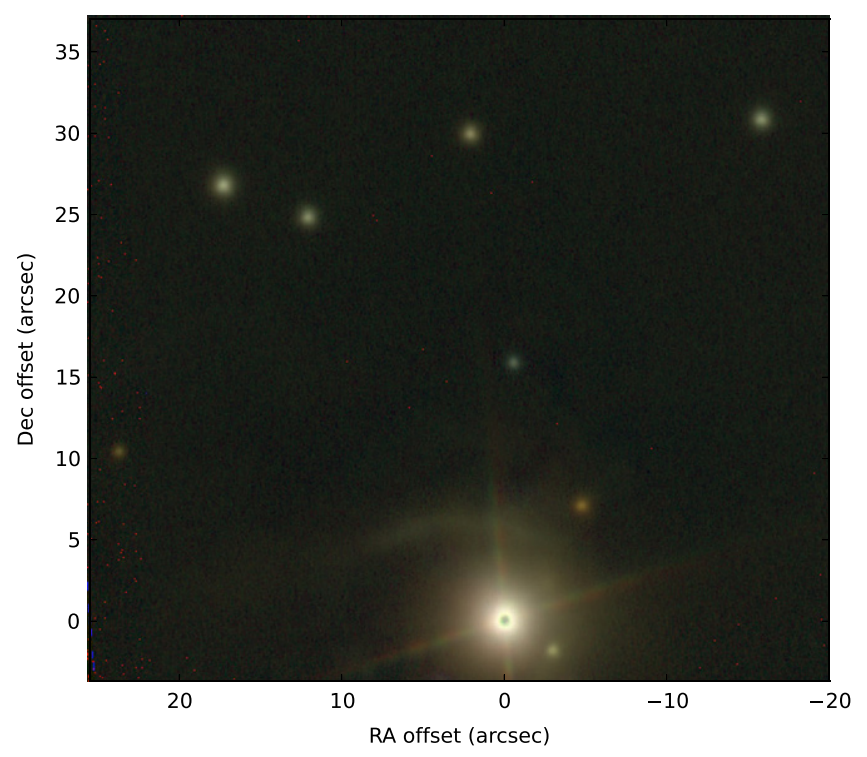

Figure 2. Near-infrared false-color composite image of a region north of V1818 Ori (located at position 0,0$)$ in the $J$ (blue), $H$ (green), and $K^{\prime}$ (red) bands observed by IRCS at the Subaru Telescope.

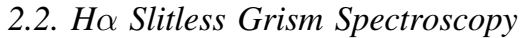

To survey $\mathrm{H} \alpha$ emission stars in the region around $\mathrm{V} 1818$ Ori, we obtained optical slitless spectroscopy using the Wide Field Grism Spectrograph 2 (WFGS2; Uehara et al. 2004) installed at the $f / 10$ Cassegrain focus of the University of Hawaii $2.2 \mathrm{~m}$ telescope on Mauna Kea. The Tektronix $2048 \times 2048$ CCD camera was used, providing a pixel scale of $0,34 \mathrm{pixel}^{-1}$. The field of view is $11^{\prime \prime} .5$ on a side and we centered the field at V1818 Ori. The observations were carried out on the night of UT 2012 February 21. The low-dispersion grism with 300 grooves $\mathrm{mm}^{-1}$ centered on $\mathrm{H} \alpha$ was used for a dispersion of $0.38 \mathrm{~nm}_{\text {pixel }}^{-1}$. Three $900 \mathrm{~s}$ exposures were obtained with a wide $\mathrm{H} \alpha$ filter $(651.5 \pm 24 \mathrm{~nm})$. Direct images were also taken with the $r^{\prime}$ and $i^{\prime}$ filters. The data were reduced using standard procedures in IRAF, and dome flats were used for flat fielding.

\subsection{High-dispersion Optical Spectroscopy}

Optical spectra of V1818 Ori and IRAS 05510-1025 were acquired on UT 2011 December 18 and UT 2013 December 27, respectively, using the High Resolution Echelle Spectrometer (HIRES; Vogt et al. 1994) at the Keck I telescope. The C1 decker $(0 . / 86 \times 7 ! .0)$ with a projected slit width of 3 pixels was used, providing a spectral resolution $R \sim 45,000$. A three-chip mosaic CCD was used in $2 \times 1$ binning format. The exposure time for V1818 Ori was 1200 s. For V1818 Ori, near complete spectral coverage in the wavelength range from $4685 \AA$ to $8724 \AA$ was obtained, with some order gaps in between. Spectra of IRAS 05510-1025 has slightly longer wavelengths with a range from 4780 to $9200 \AA$.

The data reduction of V1818 Ori was done using the standard tools in IRAF. The overscan region of the image was used to estimate the bias level. The L.A.Cosmic package (van Dokkum 2001) was used to remove cosmic rays. We examined the image to verify that cosmic rays were removed properly, and some residual cosmic rays were removed manually. The image was divided by the flat frame, and then the spectra were extracted using the apextract package in IRAF. The 
Table 1

Photometry of IRCS Sources

\begin{tabular}{|c|c|c|c|c|c|c|c|c|}
\hline $\begin{array}{l}\text { R.A. (J2000) } \\
\qquad(\mathrm{h} \mathrm{m} \mathrm{s})\end{array}$ & $\begin{array}{c}\text { Decl. (J2000) } \\
\left({ }^{\prime \prime \prime \prime)}\right)\end{array}$ & $\begin{array}{c}\text { 2MASS } \\
\text { Designation }\end{array}$ & $\begin{array}{c}J \\
(\mathrm{mag})\end{array}$ & $\begin{array}{c}\sigma_{J} \\
(\mathrm{mag})\end{array}$ & $\begin{array}{c}H \\
(\mathrm{mag})\end{array}$ & $\begin{array}{c}\sigma_{H} \\
(\mathrm{mag})\end{array}$ & $\begin{array}{c}K^{\prime} \\
(\mathrm{mag})\end{array}$ & $\begin{array}{c}\sigma_{K^{\prime}} \\
(\mathrm{mag})\end{array}$ \\
\hline 55343.7 & -102334 & 05534372-1023338 & 14.30 & 0.06 & 13.09 & 0.07 & 12.59 & 0.11 \\
\hline 55343.4 & -102336 & 05534336-1023358 & 15.09 & 0.06 & 13.99 & 0.07 & 13.57 & 0.11 \\
\hline 55342.7 & -102331 & $\ldots$ & 15.46 & 0.07 & 14.21 & 0.07 & 13.50 & 0.11 \\
\hline 55341.5 & -102330 & 05534146-1023297 & 14.85 & 0.06 & 13.78 & 0.07 & 13.41 & 0.11 \\
\hline 55342.5 & -102345 & $\ldots$ & 16.18 & 0.07 & 15.74 & 0.10 & 15.62 & 0.11 \\
\hline 55342.2 & -102354 & $05534225-1023535$ & 17.37 & 0.13 & 15.14 & 0.10 & 13.67 & 0.13 \\
\hline 55344.1 & -102350 & 05534413-1023504 & 17.98 & 0.09 & 16.23 & 0.07 & 15.09 & 0.11 \\
\hline 55342.5 & -102404 & $\ldots$ & 15.29 & 0.26 & 13.72 & 0.21 & 13.09 & 0.33 \\
\hline
\end{tabular}

Note. The photometry is done in the MKO filter system.

wavelength calibration was done using the thorium-argon lamp observations as reference and the onedspec package in IRAF. The spectra were corrected to the heliocentric frame of reference as calculated by the rvcorrect task. We did not perform absolute flux calibration; instead, we fitted the continuum level at each order and normalized the continuum to unity. The spectra of the IRAS 05510-1025 pair were reduced using $\mathrm{MAKEE}^{5}$ which produces a wavelength solution in the heliocentric frame.

\section{RESULTS AND DISCUSSION}

\subsection{Optical Spectral Properties of V1818 Ori}

Using low resolution optical spectra, Vieira et al. (2003) listed a spectral type for V1818 Ori as B7. We follow the spectral classification scheme in Hillenbrand (1995, chapter 4.2) and visually compared our HIRES spectrum with the standard spectra of the catalogs of Silva \& Cornell (1992), Pickles (1998), and the ELODIE library (Le Borgne et al. 2004). In particular, the $\mathrm{He}_{\mathrm{I}}(5876,6678,7065 \AA)$ and He II $(5412 \AA)$ lines are the most useful, although both the $\mathrm{H}$ and $\mathrm{He}$ lines can have emission components in young early type stars. Our HIRES spectrum is consistent with a B7 V star $\left(T_{\text {eff }} \sim 13,000 \mathrm{~K}\right)$. In contrast, Connelley \& Greene (2010) classified V1818 Ori as an F0 star by fitting a near-infrared spectrum with the SpeX near-infrared spectral library. However, there are no B- and A-class stellar spectra in this comparison library, and therefore a B-star classification is not excluded by Connelley \& Greene (2010). Also, the object has significant veiling which fills in the Balmer lines at nearinfrared wavelengths. We use a spectral type of $\mathrm{B} 7 \mathrm{~V}$ for V1818 Ori in this study, in agreement with Vieira et al. (2003).

To estimate the radial velocity of the star, we perform a simple Gaussian fitting to 31 unblended narrow photospheric lines, including $\mathrm{Fe}$ I, Fe II, and $\mathrm{Si}$ II, with the rest wavelengths from the NIST Atomic Spectra Database. ${ }^{6}$ The mean radial velocity is measured to be 28.5 (heliocentric) or $9.7 \mathrm{~km} \mathrm{~s}^{-1}$ (local standard of rest) with a standard deviation of $2.1 \mathrm{~km} \mathrm{~s}^{-1}$, and this is used as the rest frame velocity of the star.

The most prominent line in our spectrum is the $\mathrm{H} \alpha$ line, shown in Figure 3(a). A broad line is seen over ranges $\sim \pm 400 \mathrm{~km} \mathrm{~s}^{-1}$ from line center with an asymmetric triplepeaked feature, likely due to a combination of wind and accretion. The peaks are at $-94,+41$, and $+128 \mathrm{~km} \mathrm{~s}^{-1}$ in the

\footnotetext{
5 http://www2.keck.hawaii.edu/inst/common/makeewww/

6 http://www.nist.gov/pml/data/asd.cfm
}

stellar rest frame. The deep blueshifted wind absorption has the minimum at $-18 \mathrm{~km} \mathrm{~s}^{-1}$, indicating mass ejection. The shallower trough has the minimum at $+76 \mathrm{~km} \mathrm{~s}^{-1}$, likely from infall. Corresponding crests and troughs are also seen at the $\mathrm{H} \beta$ line profile. The $\mathrm{H} \alpha$ emission indicates a type IIB profile using the classification scheme of Reipurth et al. (1996). The broad $\mathrm{H} \alpha$ profile can be produced by the infalling gas channeled onto the stellar surface in the magnetospheric accretion model (e.g., Muzerolle et al. 2004). The line profile of the sodium resonance doublet Na I D 5890/5896 is shown in Figure 3(b); no prominent redshifted absorption is seen. Two of the Ca II triplet lines (8498 and 8662) are within the observed spectral range and strong emission is detected; however, the lines are superposed on broad Paschen absorption lines. The spectral profile around $\mathrm{Ca}$ II 8662 is shown in Figure 3(c). In addition, very weak forbidden emission lines of [O I] 6300/6363 and [S II] $6717 / 6731$ are detected, which are commonly seen in Herbig $\mathrm{Ae} / \mathrm{Be}$ stars and may indicate outflow activity (e.g., Böhm \& Catala 1994; Acke et al. 2005). The lines are too weak to measure meaningful line ratios. An attempt to measure their velocities suggest that the lines share the velocity of the star. The He I 5876, He I 6678, O I 6156 triplet, O I 7774 triplet, and O I 8446 triplet appear in absorption. The Li I 6707 line is not detected.

\subsection{Light Curve of V1818 Ori}

Herbig Ae/Be stars are generally irregularly variable, with the A stars being significantly more variable than the B stars (Herbst \& Shevchenko 1999). The All Sky Automated Survey (ASAS; Pojmanski 1997, 2002) $V$-band light curve presented in Figure 4 shows that V1818 Ori exhibits the same kind of highly irregular high-amplitude (exceeding $3 \mathrm{mag}$ ) variability that is characteristic of the UX Orionis-type subset of Herbig $\mathrm{Ae} / \mathrm{Be}$ stars. UX Ori stars tend to become bluer during their deep minima, which we cannot confirm for V1818 Ori, since we only have one color, so we can only state that it is a likely member of the UX Ori class, albeit to be confirmed. From the light curve of V1818 Ori, it is unclear if we are mainly witnessing spikes in brightening (Herbst \& Shevchenko 1999; Rucinski et al. 2010) due to accretion events, or a series of "Algol-type" deep minima due to orbiting dust (Grinin et al. 2008), or a combination of the two.

\subsection{Distance to the V1818 Ori Complex}

V1818 Ori is located near the southeastern edge of the Orion A cloud (L1641), which in projection places it adjacent to the 

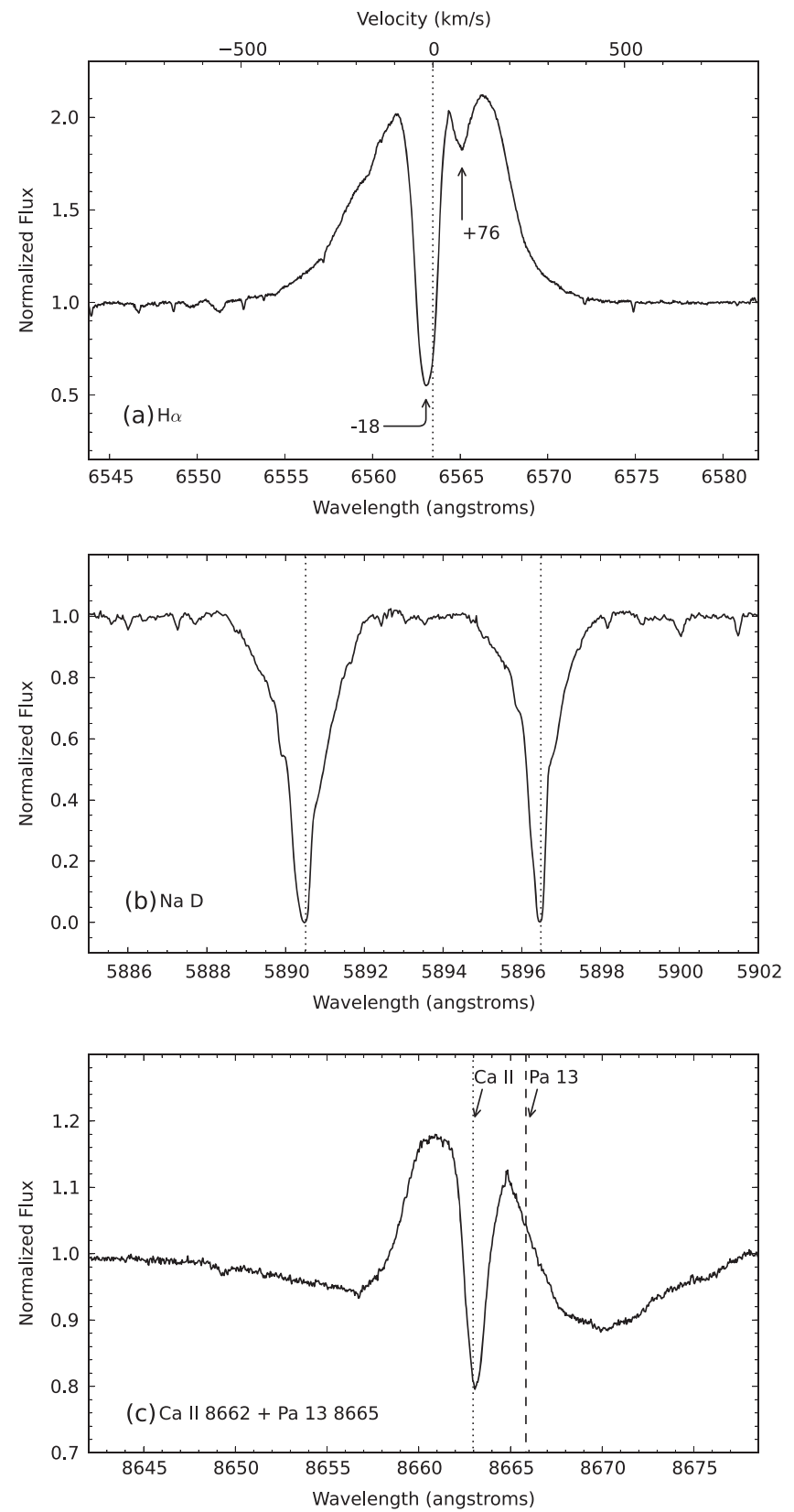

Figure 3. High-resolution spectra of (a) $\mathrm{H} \alpha$, (b) Na I doublet, and (c) Ca II blended with Paschen 13 lines obtained with HIRES at the Keck I telescope. The vertical lines show the line wavelengths redshifted to the stellar rest frame.
Table 2

$\mathrm{H} \alpha$ Emission Stars

\begin{tabular}{lccc}
\hline \hline $\begin{array}{l}\text { 2MASS } \\
\text { Designation }\end{array}$ & $\begin{array}{c}\text { R.A. (J2000) } \\
(\mathrm{h} \mathrm{m} \mathrm{s})\end{array}$ & $\begin{array}{c}\text { Decl. (J2000) } \\
\left({ }^{\circ} / \prime\right)\end{array}$ & $\begin{array}{c}\mathrm{W}(\mathrm{H} \alpha)^{\mathrm{a}} \\
(\AA)\end{array}$ \\
\hline $05534254-1024006$ & 55342.5 & -102401 & $-43^{\mathrm{b}}$ \\
$05533378-1022474$ & 55333.8 & -102247 & $-17^{\mathrm{c}}$ \\
$05534297-1026179$ & 55343.0 & -102618 & $-16^{\mathrm{c}}$ \\
$05534559-1024510$ & 55345.6 & -102451 & $-60^{\mathrm{c}}$ \\
\hline
\end{tabular}

a Negative values denote emission.

b V1818 Ori; Lee \& Chen (2009).

${ }^{\mathrm{c}}$ WFGS2 Observations.

Mon R2 and NGC 2149 regions (van den Bergh 1966). The Mon R2 reflection nebula, associated with a large group of molecular clouds east of the Orion A cloud, is a massive starforming region and contains an ultra-compact $\mathrm{H}$ II region near its core (Carpenter \& Hodapp 2008). The NGC 2149 reflection nebula is a small poorly studied star-forming region lying in projection between the Mon R2 and the Orion A cloud (Wilson et al. 2005). In this vicinity there are clouds of two distinct velocities along the line of sight $\left(V_{\mathrm{LSR}} \sim 5.8\right.$ and $\sim 10.3 \mathrm{~km} \mathrm{~s}^{-1}$, Maddalena et al. 1986). Maddalena et al. (1986) suggested that the high-velocity component is associated with the Mon R2 cloud at a much larger distance $(830 \pm 50$ pc by Herbst \& Racine 1976 and $905 \pm 37$ pc by Lombardi et al. 2011) than the Orion Nebula Cluster ( $414 \pm 7$ pc by Menten et al. 2007 and $418 \pm 6$ pc by Kim et al. 2008, also see the discussions in Muench et al. 2008). In the ${ }^{13} \mathrm{CO}$ gas emission, Kim et al. (2004) detected a $\sim 1000 M_{\odot}$. cloud clump (\#69) toward the V1818 Ori cloud complex, and its similar velocity and proximity to NGC 2149 suggest that the V1818 Ori complex is likely associated with NGC 2149 (Lee \& Chen 2009). The ${ }^{13} \mathrm{CO}$ detection is consistent with observations of the $\mathrm{CO}$ gas, which also shows an emission clump around the V1818 complex at $V_{\mathrm{LSR}} \sim 10 \mathrm{~km} \mathrm{~s}^{-1}$ (Wilson et al. 2005). Using photospheric lines, we determine the V1818 Ori radial velocity (LSR) to be $\sim 9.7 \mathrm{~km} \mathrm{~s}^{-1}$ (Section 3.1), which is similar to the LSR-velocity of $10.2 \mathrm{~km} \mathrm{~s}^{-1}$ for clump \#69 (Kim et al. 2004). Our velocity measurement thus suggests that the clump detected in the molecular lines indeed traces gas around V1818 Ori. Although Wilson et al. (2005) interpreted the velocity distribution of the NGC 2149 region as an expanding ring at a distance of only $400 \mathrm{pc}$, we favor the interpretation that the V1818 Ori complex is associated with NGC 2149 and

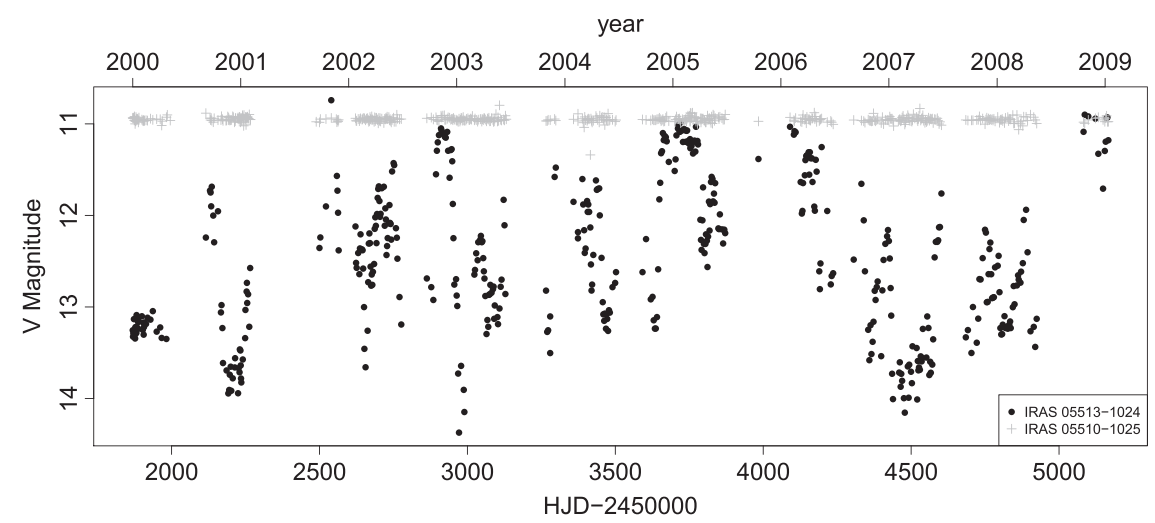

Figure 4. V-band brightness variation of V1818 Ori (IRAS 05513-1024, black dots) and IRAS 05510-1025 (gray crosses) as measured by ASAS. 
Table 3

V1818 Ori, IRAS 05510-1025, and YSO Candidates in the Vicinity

\begin{tabular}{|c|c|c|c|c|c|c|c|c|c|c|c|c|c|}
\hline ID & $\begin{array}{c}\text { 2MASS } \\
\text { Designation }\end{array}$ & $\begin{array}{l}\text { R.A. } \\
\text { (J2000) } \\
(\mathrm{h} \mathrm{m} \mathrm{s})\end{array}$ & $\begin{array}{c}\text { Decl. } \\
(\mathrm{J} 2000) \\
\left({ }^{\prime \prime \prime \prime}\right)\end{array}$ & $\begin{array}{c}\text { Distance }^{\mathrm{a}} \\
\left({ }^{\prime}\right)\end{array}$ & $\begin{array}{c}J \\
(\mathrm{mag})\end{array}$ & $\begin{array}{c}H \\
(\mathrm{mag})\end{array}$ & $\begin{array}{c}K s \\
(\mathrm{mag})\end{array}$ & $\begin{array}{c}\text { W1 } \\
(\mathrm{mag})\end{array}$ & $\begin{array}{c}W 2 \\
(\mathrm{mag})\end{array}$ & $\begin{array}{c}\text { W3 } \\
(\mathrm{mag})\end{array}$ & $\begin{array}{c}\text { W4 } \\
(\mathrm{mag})\end{array}$ & $\begin{array}{c}\text { YSO } \\
\text { Criteria }^{\mathrm{b}}\end{array}$ & $\begin{array}{c}L^{\mathrm{c}} \\
(L \odot)\end{array}$ \\
\hline V1818 Ori & 05534254-1024006 & 55342.5 & -102401 & $\cdots$ & $\begin{array}{c}9.803 \\
\pm 0.027\end{array}$ & $\begin{array}{c}7.635 \\
\pm 0.038\end{array}$ & $\begin{array}{c}5.956 \\
\pm 0.020\end{array}$ & $\begin{array}{c}4.104 \\
\pm 0.080\end{array}$ & $\begin{array}{c}2.606 \\
\pm 0.053\end{array}$ & $\begin{array}{c}0.424 \\
\pm 0.012\end{array}$ & $\begin{array}{l}-1.063 \\
\pm 0.006\end{array}$ & & 651 \\
\hline IRAS 05510-1025 & 05532989-1024337 & 55329.9 & -102433 & 3.21 & $\begin{array}{c}10.239 \\
\pm 0.047\end{array}$ & $\begin{array}{c}9.907 \\
\pm 0.049\end{array}$ & $\begin{array}{c}9.723 \\
\pm 0.033\end{array}$ & $\begin{array}{c}8.971 \\
\pm 0.021\end{array}$ & $\begin{array}{c}8.499 \\
\pm 0.020\end{array}$ & $\begin{array}{c}4.949 \\
\pm 0.016\end{array}$ & $\begin{array}{c}2.823 \\
\pm 0.023\end{array}$ & & 28.6 \\
\hline \multicolumn{14}{|c|}{ Nearby YSO Candidates } \\
\hline 1 & $05532408-1025353$ & 55324.1 & -102535 & 4.75 & $\begin{array}{c}17.03 \\
\pm 0.18\end{array}$ & $\begin{array}{c}15.74 \\
\pm 0.13\end{array}$ & $\begin{array}{c}14.72 \\
\pm 0.10\end{array}$ & $\begin{array}{c}13.35 \\
\pm 0.03\end{array}$ & $\begin{array}{c}12.82 \\
\pm 0.03\end{array}$ & $\begin{array}{l}11.43 \\
\pm 0.25\end{array}$ & $7.69 \pm 0.14$ & $\mathrm{~b}$ & 0.2 \\
\hline 2 & 05532505-1027302 & 55325.1 & -102730 & 5.49 & $\begin{array}{c}13.13 \\
\pm 0.02\end{array}$ & $\begin{array}{c}11.78 \\
\pm 0.02\end{array}$ & $\begin{array}{c}10.97 \\
\pm 0.02\end{array}$ & $\begin{array}{c}10.13 \\
\pm 0.02\end{array}$ & $\begin{array}{c}9.37 \\
\pm 0.02\end{array}$ & $\begin{array}{c}6.91 \\
\pm 0.02\end{array}$ & $4.76 \pm 0.02$ & $\mathrm{~b}$ & 5.0 \\
\hline 3 & 05532538-1027155 & 55325.4 & -102716 & 5.27 & $\begin{array}{c}13.89 \\
\pm 0.04\end{array}$ & $\begin{array}{l}12.42 \\
\pm 0.03\end{array}$ & $\begin{array}{c}11.66 \\
\pm 0.02\end{array}$ & $\begin{array}{l}11.15 \\
\pm 0.02\end{array}$ & $\begin{array}{l}10.49 \\
\pm 0.02\end{array}$ & $\begin{array}{c}8.73 \\
\pm 0.03\end{array}$ & $7.19 \pm 0.09$ & $\mathrm{~b}$ & 2.4 \\
\hline 4 & 05532749-1021254 & 55327.5 & -102126 & 4.48 & $\begin{array}{c}16.95 \\
\pm 0.17\end{array}$ & $\begin{array}{c}15.03 \\
\pm 0.09\end{array}$ & $\begin{array}{c}14.20 \\
\pm 0.07\end{array}$ & $\begin{array}{c}13.79 \\
\pm 0.03\end{array}$ & $\begin{array}{c}13.34 \\
\pm 0.03\end{array}$ & $\begin{array}{c}11.67 \\
\pm 0.19\end{array}$ & $8.54 \pm 0.26$ & $\mathrm{~b}$ & 1.0 \\
\hline 5 & 05533303-1027128 & 55333.0 & -102713 & 3.92 & $\begin{array}{l}15.65 \\
\pm 0.08\end{array}$ & $\begin{array}{c}13.62 \\
\pm 0.05\end{array}$ & $\begin{array}{c}12.37 \\
\pm 0.03\end{array}$ & $\begin{array}{c}11.23 \\
\pm 0.02\end{array}$ & $\begin{array}{c}10.39 \\
\pm 0.02\end{array}$ & $\begin{array}{c}8.30 \\
\pm 0.02\end{array}$ & $5.50 \pm 0.03$ & $\mathrm{a}, \mathrm{b}$ & 2.1 \\
\hline 6 & 05533371-1019576 & 55333.7 & -101958 & 4.58 & $\begin{array}{c}16.29 \\
\pm 0.09\end{array}$ & $\begin{array}{c}15.53 \\
\pm 0.12\end{array}$ & $\begin{array}{c}14.88 \\
\pm 0.12\end{array}$ & NA & NA & NA & NA & $\mathrm{a}$ & \\
\hline 7 & 05533378-1022474 & 55333.8 & -102247 & 2.44 & $\begin{array}{c}13.42 \\
\pm 0.03\end{array}$ & $\begin{array}{c}12.50 \\
\pm 0.03\end{array}$ & $\begin{array}{c}12.04 \\
\pm 0.03\end{array}$ & $\begin{array}{c}11.20 \\
\pm 0.02\end{array}$ & $\begin{array}{c}10.59 \\
\pm 0.02\end{array}$ & $\begin{array}{c}8.60 \\
\pm 0.03\end{array}$ & $6.76 \pm 0.09$ & $\mathrm{~b}, \mathrm{c}$ & 5.4 \\
\hline 8 & 05533402-1026146 & 55334.0 & -102615 & 3.02 & $\begin{array}{c}13.59 \\
\pm 0.02\end{array}$ & $\begin{array}{c}13.08 \\
\pm 0.03\end{array}$ & $\begin{array}{c}12.87 \\
\pm 0.03\end{array}$ & $\begin{array}{c}12.80 \\
\pm 0.03\end{array}$ & $\begin{array}{c}12.46 \\
\pm 0.03\end{array}$ & $\begin{array}{c}10.46 \\
\pm 0.12\end{array}$ & $7.42 \pm 0.14$ & $\mathrm{~b}$ & 0.3 \\
\hline 9 & 05533457-1021226 & 55334.6 & -102123 & 3.26 & $\begin{array}{c}14.57 \\
\pm 0.03\end{array}$ & $\begin{array}{c}13.66 \\
\pm 0.03\end{array}$ & $\begin{array}{c}13.35 \\
\pm 0.04\end{array}$ & $\begin{array}{c}12.97 \\
\pm 0.03\end{array}$ & $\begin{array}{c}12.50 \\
\pm 0.03\end{array}$ & $\begin{array}{c}10.46 \\
\pm 0.08\end{array}$ & $8.07 \pm 0.20$ & $\mathrm{~b}$ & 0.3 \\
\hline 10 & 05533686-1026411 & 55336.9 & -102641 & 2.98 & $\begin{array}{c}15.93 \\
\pm 0.07\end{array}$ & $\begin{array}{c}15.30 \\
\pm 0.09\end{array}$ & $\begin{array}{c}14.80 \\
\pm 0.11\end{array}$ & $\begin{array}{c}14.85 \\
\pm 0.04\end{array}$ & $\begin{array}{l}14.48 \\
\pm 0.06\end{array}$ & $<12.50$ & $8.80 \pm 0.37$ & a & 0.1 \\
\hline 11 & 05533796-1020515 & 55338.0 & -102052 & 3.34 & $\begin{array}{c}15.71 \\
\pm 0.08\end{array}$ & $\begin{array}{c}14.61 \\
\pm 0.10\end{array}$ & $\begin{array}{c}13.83 \\
\pm 0.07\end{array}$ & $\begin{array}{c}12.81 \\
\pm 0.03\end{array}$ & $\begin{array}{c}12.53 \\
\pm 0.03\end{array}$ & $\begin{array}{c}9.44 \\
\pm 0.04\end{array}$ & $7.49 \pm 0.11$ & a & 0.4 \\
\hline 12 & 05533991-1023508 & 55339.9 & -102351 & 0.62 & $\begin{array}{c}13.09 \\
\pm 0.03\end{array}$ & $\begin{array}{c}11.59 \\
\pm 0.03\end{array}$ & $\begin{array}{c}10.60 \\
\pm 0.02\end{array}$ & $\begin{array}{c}9.70 \\
\pm 0.02\end{array}$ & $\begin{array}{c}9.07 \\
\pm 0.02\end{array}$ & $\begin{array}{c}7.97 \\
\pm 0.03\end{array}$ & $4.73 \pm 0.05$ & $\mathrm{a}, \mathrm{b}$ & 9.0 \\
\hline 13 & 05534054-1024033 & 55340.5 & -102403 & 0.44 & $\begin{array}{c}12.61 \\
\pm 0.03\end{array}$ & $\begin{array}{l}11.12 \\
\pm 0.02\end{array}$ & $\begin{array}{c}10.08 \\
\pm 0.02\end{array}$ & $\begin{array}{c}8.31 \\
\pm 0.02\end{array}$ & $\begin{array}{c}7.54 \\
\pm 0.02\end{array}$ & $\begin{array}{c}5.26 \\
\pm 0.02\end{array}$ & $2.99 \pm 0.02$ & $\mathrm{a}, \mathrm{b}$ & 155 \\
\hline 14 & 05534225-1023535 & 55342.3 & -102354 & 0.13 & $\begin{array}{c}17.50 \\
\pm 0.14\end{array}$ & $\begin{array}{l}15.10 \\
\pm 0.10\end{array}$ & $\begin{array}{c}13.75 \\
\pm 0.13\end{array}$ & NA & NA & NA & NA & $\mathrm{d}$ & \\
\hline 15 & 05534297-1026179 & 55343.0 & -102618 & 2.28 & $\begin{array}{c}13.05 \\
\pm 0.02\end{array}$ & $\begin{array}{c}12.04 \\
\pm 0.02\end{array}$ & $\begin{array}{l}11.57 \\
\pm 0.02\end{array}$ & $\begin{array}{c}10.85 \\
\pm 0.03\end{array}$ & $\begin{array}{c}10.23 \\
\pm 0.02\end{array}$ & $\begin{array}{c}7.93 \\
\pm 0.02\end{array}$ & $5.66 \pm 0.04$ & $\mathrm{~b}, \mathrm{c}$ & 1.3 \\
\hline 16 & 05534413-1023504 & 55344.1 & -102350 & 0.48 & $\begin{array}{c}18.08 \\
\pm 0.09\end{array}$ & $\begin{array}{c}16.21 \\
\pm 0.07\end{array}$ & $\begin{array}{l}15.15 \\
\pm 0.11\end{array}$ & NA & NA & NA & NA & $\mathrm{d}$ & \\
\hline 17 & 05534500-1023217 & 55345.0 & -102322 & 0.93 & $\begin{array}{c}14.10 \\
\pm 0.03\end{array}$ & $\begin{array}{c}12.71 \\
\pm 0.03\end{array}$ & $\begin{array}{c}11.51 \\
\pm 0.02\end{array}$ & $\begin{array}{c}10.85 \\
\pm 0.02\end{array}$ & $\begin{array}{c}10.44 \\
\pm 0.02\end{array}$ & $\begin{array}{c}8.22 \\
\pm 0.02\end{array}$ & $4.63 \pm 0.03$ & $\mathrm{a}, \mathrm{b}$ & 2.6 \\
\hline 18 & 05534559-1024510 & 55345.6 & -102451 & 1.15 & $\begin{array}{c}12.95 \\
\pm 0.02\end{array}$ & $\begin{array}{l}11.72 \\
\pm 0.02\end{array}$ & $\begin{array}{c}10.76 \\
\pm 0.02\end{array}$ & $\begin{array}{c}9.40 \\
\pm 0.02\end{array}$ & $\begin{array}{c}8.64 \\
\pm 0.02\end{array}$ & $\begin{array}{c}6.57 \\
\pm 0.02\end{array}$ & $3.71 \pm 0.02$ & $\mathrm{a}, \mathrm{b}, \mathrm{c}$ & 6.2 \\
\hline 19 & 05534767-1025060 & 55347.7 & -102506 & 1.70 & $\begin{array}{c}12.84 \\
\pm 0.03\end{array}$ & $\begin{array}{c}11.92 \\
\pm 0.02\end{array}$ & $\begin{array}{c}11.44 \\
\pm 0.02\end{array}$ & $\begin{array}{c}11.00 \\
\pm 0.02\end{array}$ & $\begin{array}{c}10.49 \\
\pm 0.02\end{array}$ & $\begin{array}{c}8.85 \\
\pm 0.03\end{array}$ & $5.56 \pm 0.04$ & $\mathrm{~b}$ & 1.3 \\
\hline 20 & 05535073-1025238 & 55350.7 & -102524 & 2.48 & & & & & & & $7.29 \pm 0.09$ & $\mathrm{~b}$ & 0.3 \\
\hline
\end{tabular}


Table 3

(Continued)

\begin{tabular}{|c|c|c|c|c|c|c|c|c|c|c|c|c|c|}
\hline ID & $\begin{array}{c}\text { 2MASS } \\
\text { Designation }\end{array}$ & $\begin{array}{l}\text { R.A. } \\
(\mathrm{J} 2000) \\
(\mathrm{h} \mathrm{m} \mathrm{s}) \\
\end{array}$ & $\begin{array}{c}\text { Decl. } \\
(\mathrm{J} 2000) \\
\left({ }^{\circ \prime \prime \prime}\right)\end{array}$ & $\begin{array}{c}\text { Distance }^{\mathrm{a}} \\
\left(^{\prime}\right) \\
\end{array}$ & $\begin{array}{c}J \\
(\mathrm{mag}) \\
\end{array}$ & $\begin{array}{c}H \\
(\mathrm{mag}) \\
\end{array}$ & $\begin{array}{c}K s \\
(\mathrm{mag})\end{array}$ & $\begin{array}{c}W 1 \\
(\mathrm{mag})\end{array}$ & $\begin{array}{c}W 2 \\
(\mathrm{mag})\end{array}$ & $\begin{array}{c}\text { W3 } \\
(\mathrm{mag})\end{array}$ & $\begin{array}{c}\text { W4 } \\
(\mathrm{mag})\end{array}$ & $\begin{array}{c}\text { YSO } \\
\text { Criteria }^{b}\end{array}$ & $\begin{array}{c}L^{\mathrm{c}} \\
(L \odot) \\
\end{array}$ \\
\hline & & & & & $\begin{array}{c}14.43 \\
\pm 0.03\end{array}$ & $\begin{array}{c}13.54 \\
\pm 0.03\end{array}$ & $\begin{array}{c}13.16 \\
\pm 0.03\end{array}$ & $\begin{array}{c}12.92 \\
\pm 0.03\end{array}$ & $\begin{array}{c}12.61 \\
\pm 0.03\end{array}$ & $\begin{array}{c}11.10 \\
\pm 0.14\end{array}$ & & & \\
\hline 21 & $05535140-1022377$ & 55351.4 & -102238 & 2.63 & $\begin{array}{c}16.41 \\
\pm 0.11\end{array}$ & $\begin{array}{c}15.26 \\
\pm 0.08\end{array}$ & $\begin{array}{c}14.81 \\
\pm 0.11\end{array}$ & $\begin{array}{c}13.83 \\
\pm 0.05\end{array}$ & $\begin{array}{c}13.24 \\
\pm 0.06\end{array}$ & $\begin{array}{l}11.28 \\
\pm 0.21\end{array}$ & $8.59 \pm 0.32$ & $\mathrm{~b}$ & 1.6 \\
\hline 22 & NA & 55402.1 & -102443 & 4.92 & NA & NA & NA & $\begin{array}{c}13.70 \\
\pm 0.03\end{array}$ & $\begin{array}{c}12.12 \\
\pm 0.02\end{array}$ & $\begin{array}{c}8.73 \\
\pm 0.02\end{array}$ & $6.59 \pm 0.06$ & $\mathrm{~b}$ & $\cdots$ \\
\hline
\end{tabular}

Note. 2MASS and WISE magitudes from the respective online catalogs. The equations in Connelley et al. (2007) were used for transforming from MKO system to 2 MASS system.

a Angular distance to V1818 Ori.

b YSO selection criteria: (a) 2MASS infrared excess; (b) WISE colors; (c) H $\alpha$ emission; (d) IRCS infrared excess.

${ }^{\mathrm{c}}$ Total luminosity of the best-fit models from Robitaille et al. (2006); see Section 3.6. 


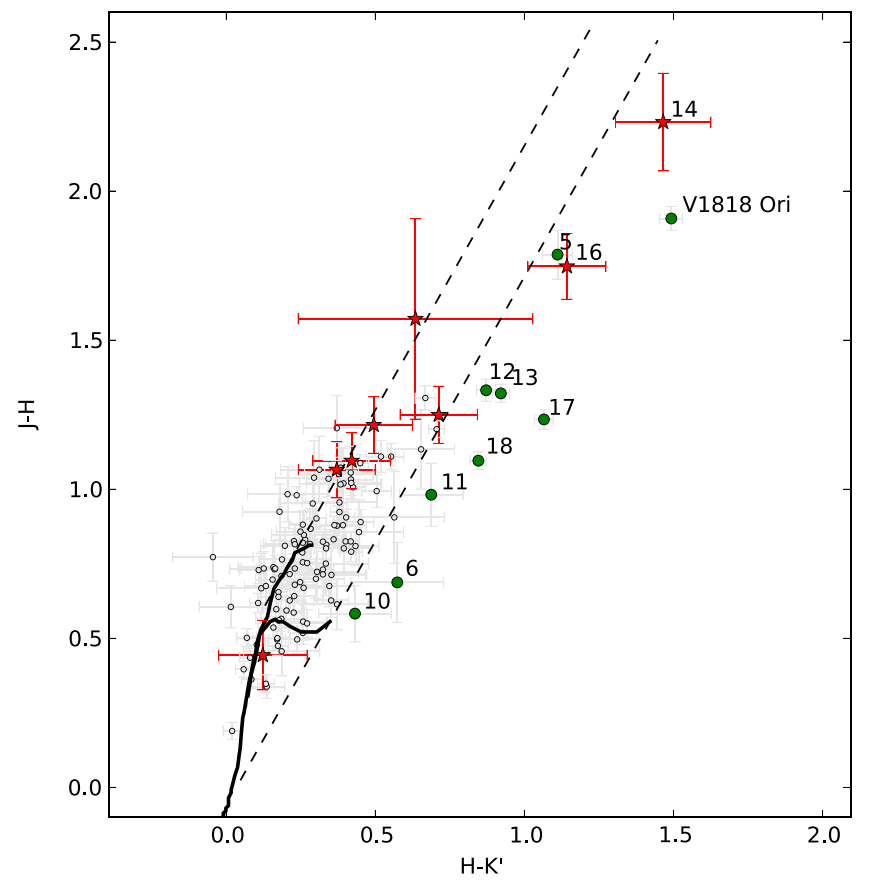

Figure 5. $J-H$ vs. $H-K^{\prime}$ color-color diagram of stars within $10^{\prime}$ of V1818 Ori. The photometry is measured in or converted to the MKO system. Red stars mark new data observed by Subaru IRCS. Open circles are field stars from the 2MASS catalog. Green filled circles are 2MASS stars with significant infrared excess; hence YSO candidates. Solid curves denote the location of main sequence dwarfs and giants, and dashed lines indicate the reddening slope. Sources that indicate infrared excess are YSO candidates and labeled with the ID numbers from Table 3.

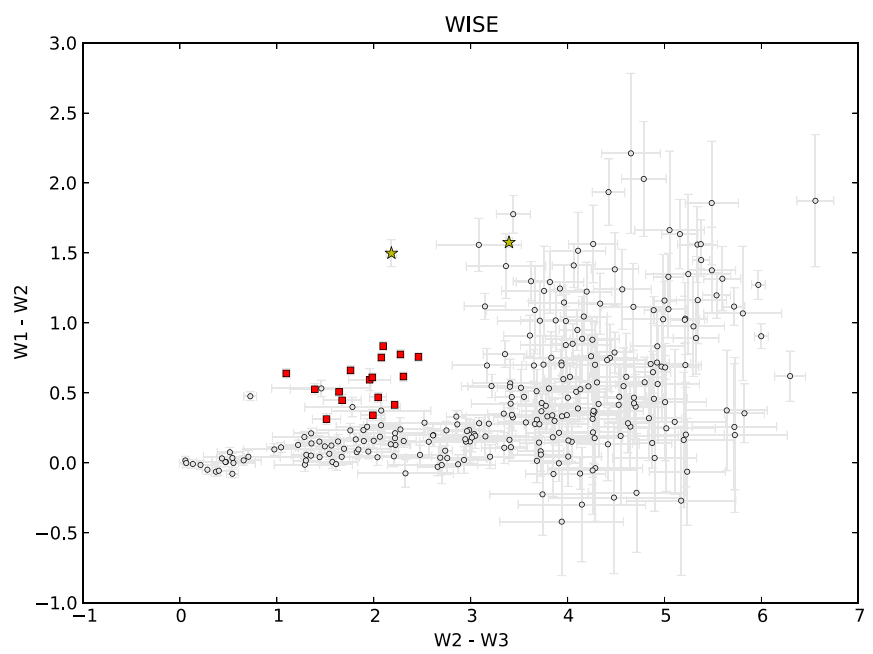

Figure 6. WISE W1-W2 vs. W2-W3 color-color diagram showing stars within a $10^{\prime}$ square box surrounding V1818 Ori. W1/W2/W3 correspond to 3.4/4.6/ $12 \mu \mathrm{m}$. Following the classification scheme of Koenig et al. (2012), the selected YSO candidates are marked as filled squares for Class II YSOs and filled pentagrams for Class I YSOs. Field objects, including plausible extragalactic objects, are shown as open circles.

the Mon R2 cloud at a larger distance. In this paper, we adopt a distance of $905 \pm 37 \mathrm{pc}$ (Lombardi et al. 2011) for V1818 Ori.

\subsection{Ho Emission Line Stars around V1818 Ori}

With the UH88 slitless grism observations, three $\mathrm{H} \alpha$ emission line stars besides V1818 Ori were detected. We identify these
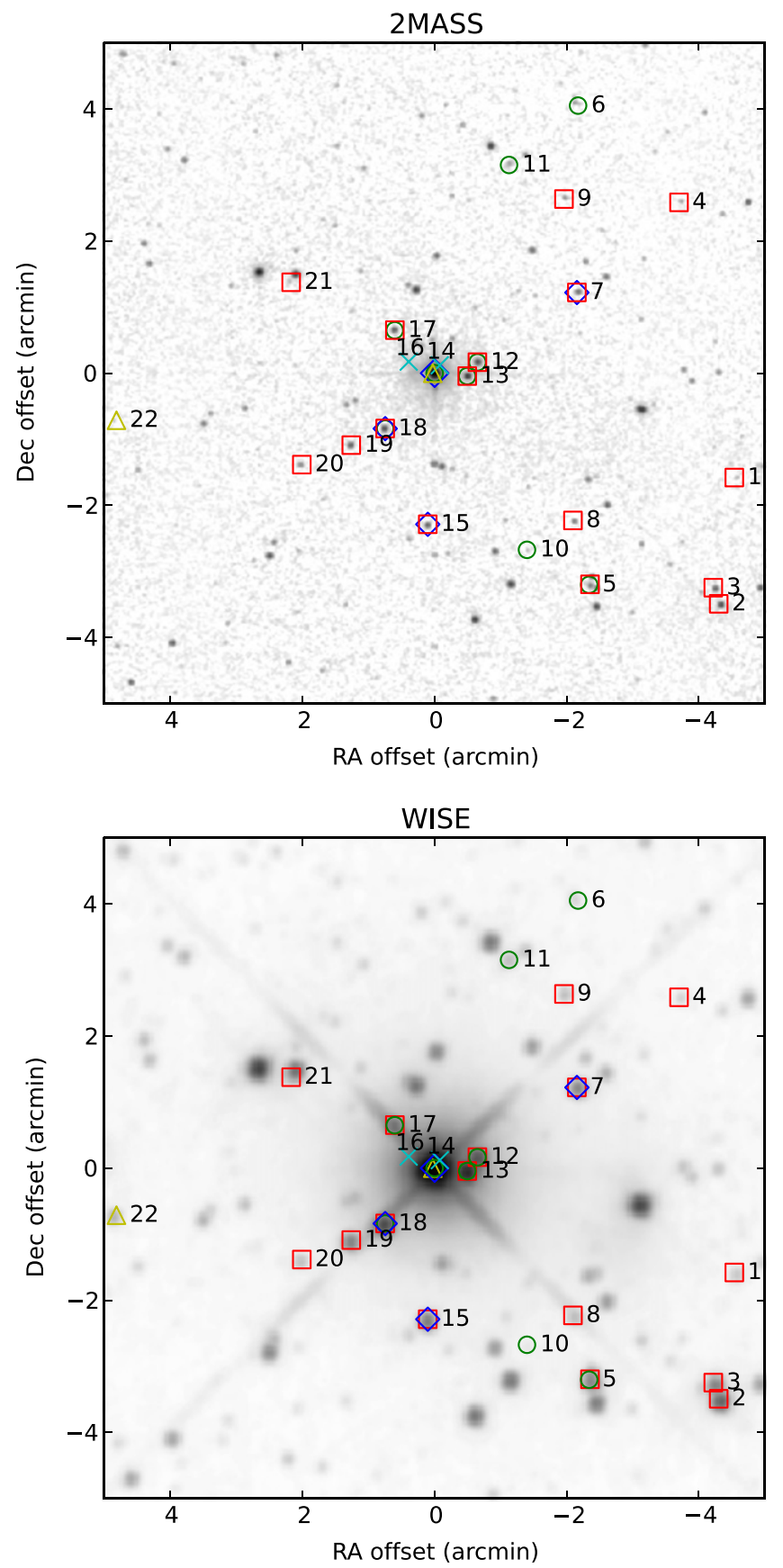

Figure 7. YSO candidates selected by various criteria overlaid on a 2MASS $K_{s}$-band $(2.2 \mu \mathrm{m})$ image (top) and a WISE W2-band $(4.6 \mu \mathrm{m})$ image (bottom) of the V1818 Ori field. Green circles mark those with 2MASS infrared excess. Blue diamonds are $\mathrm{H} \alpha$ emitters from WFGS observations. Cyan crosses indicate infrared excess from IRCS photometry. YSO candidates selected by WISE color-color diagram are marked by red squares (Class II) and yellow triangles (Class I) using the method of Koenig et al. (2012). The labels are the source ID number in Table 3.

$\mathrm{H} \alpha$ emitters in the 2MASS (Skrutskie et al. 2006) database, as given in Table 2. We measure the equivalent width of the $\mathrm{H} \alpha$ line $\mathrm{W}(\mathrm{H} \alpha)$, an indicator of accretion processes and chromospheric activity in young stars, and the results are given in Table 2. Among these objects, 2MASS J05534559-1024510 was identified as a young star by Lee \& Chen (2009). As W $(\mathrm{H} \alpha)$ of all three sources are larger than $10 \AA$, they are all likely to be Classical T Tauri stars (CTTSs; e.g., Herbig 1998). The stars are identified in Figure 7. 

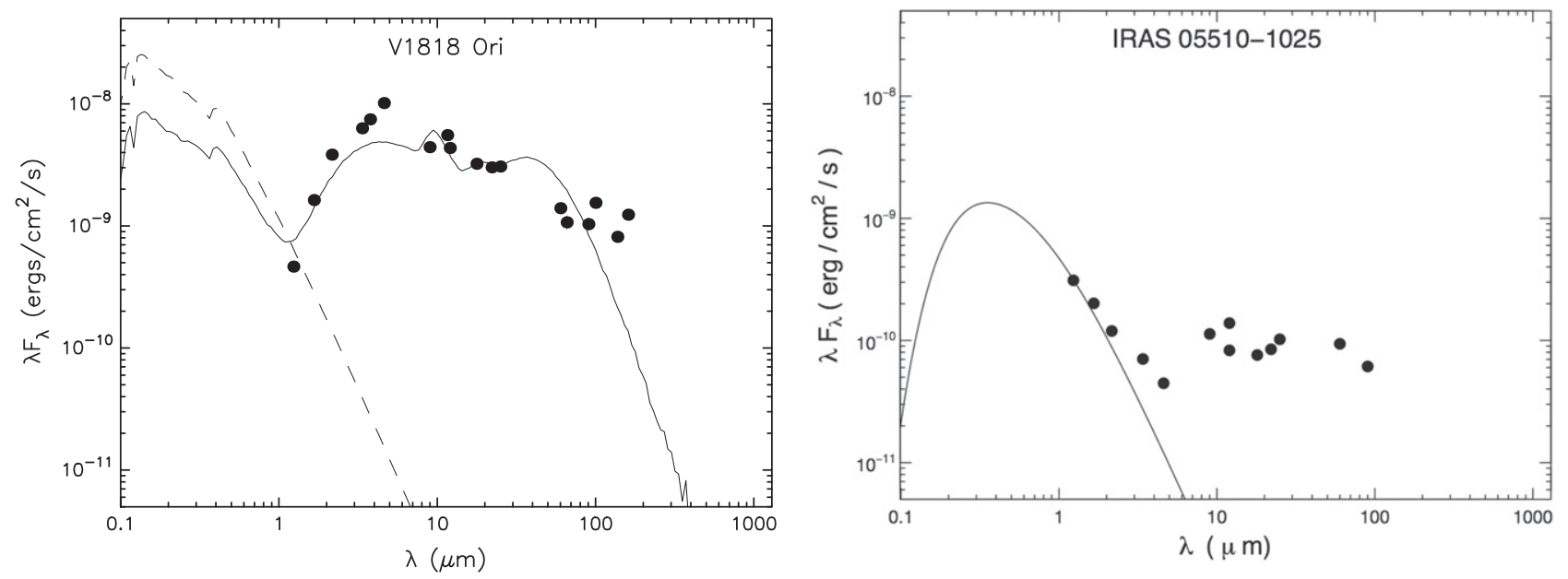

Figure 8. (Left) SED of V1818 Ori fitted with the model grid of Robitaille et al. (2006, 2007). The best fit YSO model is shown in black curve and its stellar photosphere in dashed curves. Subsequent good fits with $\chi^{2}-\chi_{\text {best }}^{2}<3$ per data point are shown in gray curves. Observational data are shown in filled circles. (Right) SED of IRAS 05510-1025. The curve represents the combined flux densities of a B9 star and a G3 star, both approximated by black bodies; their fluxes are set equal in the $J$ band, see the text.

\subsection{The Near-infrared Color-Color Diagram}

We utilize the near-infrared color-color diagram to reveal properties of stars near V1818 Ori. In Figure 5, the nearinfrared colors of the sources detected by our IRCS observations are marked by stars with the error bars as given in Table 1.

To investigate star formation activities around V1818 Ori, we arbitrarily choose a region of $10^{\prime}$ square centered on V1818 Ori and obtain the near-infrared $J / H / K_{S}$ photometry from the 2MASS All-Sky Point Source Catalog. Stars with photometric quality flag $\mathrm{C}$ or worse are excluded. For comparison between IRCS data and 2MASS data in the color-color diagram, the colors in the 2MASS $J / H / K_{S}$ system are converted to the MKO system using the updated equations of Carpenter $(2001)^{7}$ and the $K^{\prime}-K$ relation from Wainscoat \& Cowie (1992). The results are plotted in Figure 5 as circles.

In Figure 5, the solid curves mark the intrinsic colors of main sequence dwarfs and giants from Bessell \& Brett (1988) and Tokunaga \& Cox (2000). Dashed lines extend the reddening boundary from the main sequence using the reddening slope from Cohen et al. (1981). Colors are all transformed into the MKO system accordingly. Sources located on the right of the reddening line of main sequence stars indicate infrared excess and are likely to be young. We include these young stellar object (YSO) candidates in Table 3.

We further search the data release of the Wide-field Infrared Survey Explorer (WISE; Wright et al. 2010) in 3.4 (WI), 4.6 (W2), 12 (W3), and $22 \mu \mathrm{m}$ (W4) for the same $10^{\prime}$ box around V1818 Ori. We use the magnitudes from the WISE database (Wright et al. 2010). Suspicious artifact detections with contamination and confusion flags are removed, unless there are independent detections of the targets such as 2MASS measurements. Based on the WISE colors, we adopted the method of Koenig et al. (2012) to select YSO candidates. Figure 6 shows the color-color diagram in $W 1-W 2$ versus $W 2-W 3$, with the filled symbols marking the YSO candidates and the open symbols marking the background stars. Using the criteria of Koenig et al. (2012), we identified two Class I YSOs and 16 Class II YSOs.

http://www.astro.caltech.edu/ jmc/2mass/v3/transformations/
One of the Class I YSOs is V1818 Ori. The YSO candidates surrounding V1818 Ori are listed in Table 3. Figure 7 shows the spatial distribution of these young stars, selected from multiple criteria, on the 2MASS and the WISE images. It is evident that V1818 Ori joins the growing number of Herbig Ae/Be stars that are surrounded by small clusters of young low-mass stars, as originally discovered by Hillenbrand (1995) and Testi et al. (1998, 1999).

\subsection{YSO Candidates and Their Spectral Energy Distributions}

In the previous sections we use the criteria of $\mathrm{H} \alpha$ emission and infrared excess based on either $J / H / K$ or WISE colors to select YSOs. The YSO candidates are listed in Table 3. In our sample, all $\mathrm{H} \alpha$ stars show infrared excess. Figure 8 and Figure 9 show the spectral energy distributions (SEDs) of V1818 Ori, IRAS 05510-1025, and the YSO candidates with flux values available in both $J / H / K$ and WISE bands. We fit these SEDs with the grid of YSO models from Robitaille et al. (2006); good fits with $\chi^{2}-\chi_{\text {best }}^{2}<3$ per data point are shown in Figure 9 (Robitaille et al. 2007). Many of these YSOs have little circumstellar material left in the surrounding envelopes and disks. The fits are consistent with the classification results using the criteria of Koenig et al. (2012). While the energy distribution of V1818 Ori shows a major near- and far-infrared excess, the excess of IRAS 05510-1025 is much more modest.

\subsection{V1818 Ori and IRAS 05510-1025: Not a Wide Binary}

Figure 1 shows that V1818 Ori is not the only star in the field that is associated with nebulosity. About 3.2 west of V1818 Ori there is a bright star (about 2 mag brighter in $\mathrm{V}$ than V1818 Ori) associated with diffuse nebulosity. It is identified as IRAS 05510-1025 or 2MASS J05532989-1024337. Figure 4 shows the light curve of IRAS 05510-1025, which reveals no signs of variability. The obvious reflection nebulosity indicates that the star is associated with a cloud in its vicinity. The HIRES spectrum of this star shows no evidence for any emission lines, nor any line profiles indicating mass loss or infall. While placing the star on the spectrograph slit it was apparent that it is a visual binary, with a redder companion to the northeast at a separation of about $3^{\prime \prime}$. 

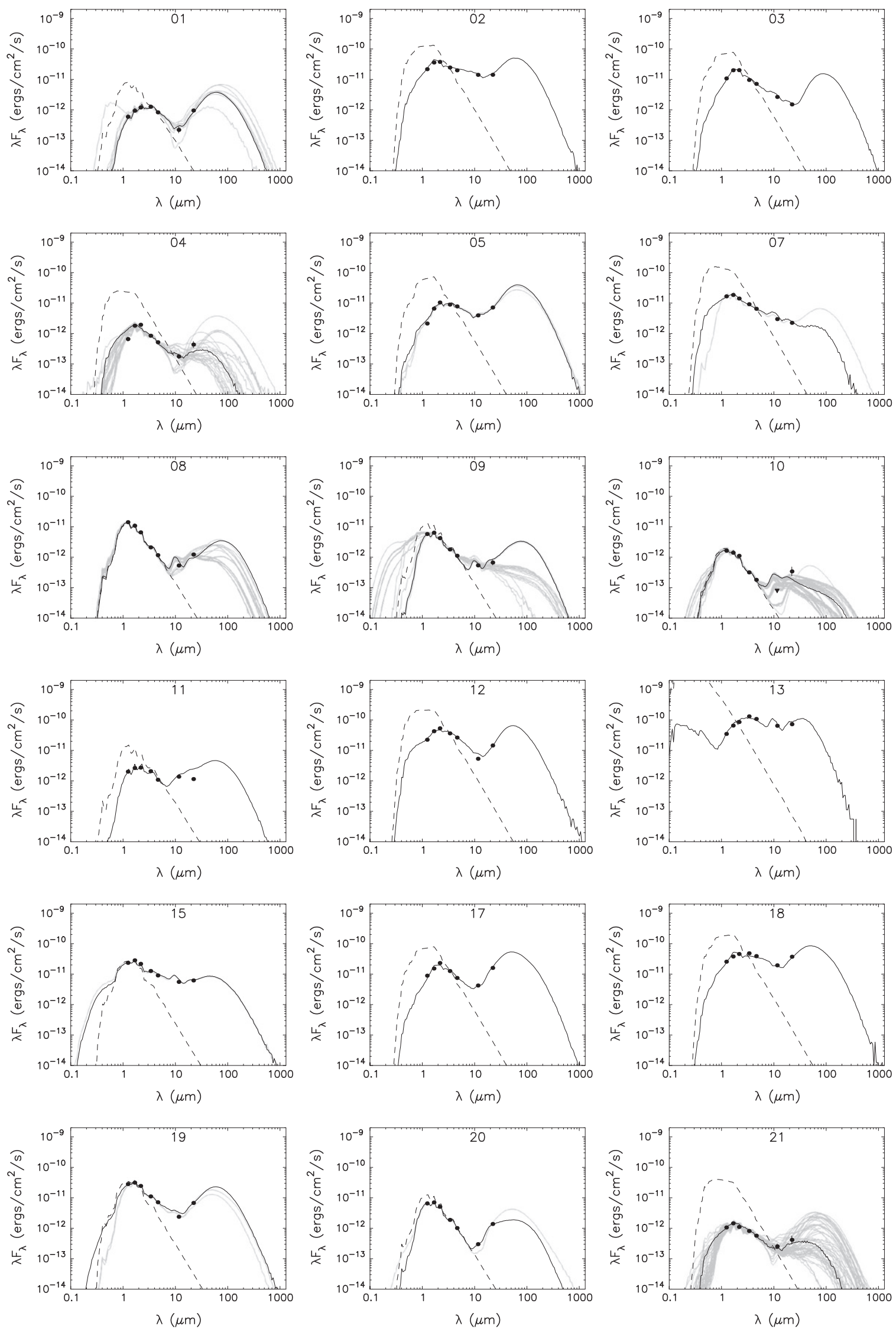

Figure 9. SEDs of YSO candidates in the vicinity of V1818 Ori fitted with the model grid of Robitaille et al. (2006, 2007). Data are shown in filled circles, the best fit YSO model in black curve and its stellar photosphere in dashed curves. Subsequent good fits with $\chi^{2}-\chi_{\text {best }}^{2}<3$ per data point are shown in gray curves. The labels are the source ID number in Table 3. 
We examined the images from DSS, 2MASS, and WISE, and found the system circular in the DSS images, elongated in the SW-NE direction in the 2MASS images, and the peak increasingly displaced toward the NE in the WISE images. The pair was placed on the slit, and despite poor seeing it was possible to extract a spectrum of both binary components. The spectral type of the NE component is F9-G5, and that of the SW component is B9. The system is either a late B main sequence and $\mathrm{G}$ giant, or a $\mathrm{B}$ subdwarf and a $\mathrm{G}$ main sequence companion.

Radial velocities for both components could be measured, the B star has $V_{\mathrm{LSR}} \sim-36.9 \pm 0.7 \mathrm{~km} \mathrm{~s}^{-1}$ and the $\mathrm{G}$ star has $V_{\mathrm{LSR}} \sim-36.0 \pm 0.8 \mathrm{~km} \mathrm{~s}^{-1}$. This is very different from the LSR-velocities of both L1641 $\left(\sim 5.8 \mathrm{~km} \mathrm{~s}^{-1}\right)$ and the more distant Monoceros cloud \#69 $\left(\sim 10.2 \mathrm{~km} \mathrm{~s}^{-1}\right)$, so evidently the binary is an interloper that is accidentally passing through either one or the other of these clouds. To estimate the distance of the pair, we can assume that the observed combined magnitude at $J$ of 10.24 is split evenly between the late B and early G star, which looks reasonable based on the 2MASS images; each component then has a $J$ magnitude of 10.99 . If one then uses $V-J=-0.09$ for the B dwarf star and $V-J=-1.52$ for the $\mathrm{G}$ star, a distance of $1200 \mathrm{pc}$ is obtained without reddening for both objects, with $M_{V}=-0.3$ for a B main sequence star and using $M_{V}=-2.1$ corresponding to a $\mathrm{G}$ subgiant or low luminosity giant. For a modest reddening $\left(A_{V} \sim 0.6\right)$, the distance would then be consistent with the $\sim 905 \mathrm{pc}$ distance of the Monoceros clouds. A higher extinction could bring the binary star as close as Orion. If V1818 Ori and the binary are in the same cloud at a distance of $\sim 905 \mathrm{pc}$, then their projected separation is $173,000 \mathrm{AU}$, or $0.85 \mathrm{pc}$.

That IRAS $05510-1025$ is an older star accidentally passing through a cloud is supported by other evidence. The binary shows little infrared excess in the 2MASS bands. But as can be seen in Figure 8, the star has a substantial excess at longer wavelengths. The clear displacement of the WISE images mentioned above suggests that at least part of the IR flux is due to the late-type companion. The excess in the energy distribution at the longest wavelengths could additionally be caused by the star heating the surface of the nearby cloud, the proximity of which is indicated by the reflection nebulosity seen in Figure 1.

\section{CONCLUDING COMMENTS}

In this paper, we focus on the Herbig Be star V1818 Ori near NGC 2149. Located along a line of sight between the Orion A cloud (L1641) and the Mon R2 cloud, the source is not included in most surveys of L1641. Wind and accretion activities at V1818 Ori are suggested by our high resolution optical spectra, expected for an intermediate-mass star in the pre-main sequence stage. In the vicinity of V1818 Ori, we identify YSO candidates using multiple criteria applied to new and archived data at optical and infrared wavelengths. A rich group of young stars is found around V1818 Ori, consistent with the findings of Hillenbrand (1995) and Testi et al. $(1998,1999)$ who both noted that small compact clusters around Herbig Ae/Be stars are common. V1818 Ori has been observed at high angular resolution with natural guide star adaptive optics by Connelley et al. (Subaru, 2008) and T. Dupuy (Keck, private communication). While the majority of Herbig $\mathrm{Ae} / \mathrm{Be}$ stars reside in binary systems (Baines et al. 2006), no close binary is detected, although a component $\sim 3 \prime \prime 5\left(\sim 1450\right.$ AU) away at a P.A. of $\sim 239^{\circ}$ from V1818 Ori is seen by both the AO observations and our IRCS observations (Figure 2).

We are grateful to the late George H. Herbig for discussions on HIRES reduction. We thank Michael S. Connelley for discussions on IR data reduction, Tae-Soo Pyo for his assistance during the IRCS observations, and Trent Dupuy for his Keck AO observations. The authors wish to recognize and acknowledge the very significant cultural role and reverence that the summit of Mauna Kea has always had within the indigenous Hawaiian community. We are most fortunate to have the opportunity to conduct observations from this mountain. Some of the data presented herein were obtained at the W.M. Keck Observatory, which is operated as a scientific partnership among the California Institute of Technology, the University of California and the National Aeronautics and Space Administration. The Observatory was made possible by the generous financial support of the W.M. Keck Foundation. This research made use of the NASA/IPAC Infrared Science Archive, which is operated by the Jet Propulsion Laboratory, California Institute of Technology, under contract with the National Aeronautics and Space Administration. This publication made use of data products from the Two Micron All Sky Survey, which is a joint project of the University of Massachusetts and the Infrared Processing and Analysis Center/California Institute of Technology, funded by the National Aeronautics and Space Administration and the National Science Foundation. This publication made use of data products from the Wide-field Infrared Survey Explorer, which is a joint project of the University of California, Los Angeles, and the Jet Propulsion Laboratory/California Institute of Technology, funded by the National Aeronautics and Space Administration. This research made use of Montage, funded by the National Aeronautics and Space Administration's Earth Science Technology Office, Computational Technologies Project, under Cooperative Agreement Number NCC5-626 between NASA and the California Institute of Technology. The code is maintained by the NASA/IPAC Infrared Science Archive. The Digitized Sky Surveys were produced at the Space Telescope Science Institute under U.S. Government grant NAG W-2166. We acknowledge support by the National Aeronautics and Space Administration through the NASA Astrobiology Institute under Cooperative Agreement No. NNA09DA77A issued through the Office of Space Science.

Facilities: $\quad$ Subaru(IRCS), Keck:I(HIRES), UH:2.2m (WFGS2)

\section{REFERENCES}

Acke, B., van den Ancker, M. E., \& Dullemond, C. P. 2005, A\&A, 436, 209 Baines, D., Oudmaijer, R. D., Porter, J. M., \& Pozzo, M. 2006, MNRAS, 367, 737

Bessell, M. S., \& Brett, J. M. 1988, PASP, 100, 1134

Böhm, T., \& Catala, C. 1994, A\&A, 290, 167

Carpenter, J. M. 2001, AJ, 121, 2851

Carpenter, J. M., \& Hodapp, K. W. 2008, in Handbook of Star Forming Regions, Vol. 1, ed. B. Reipurth (San Francisco, CA: ASP Monograph Publications), 899

Cohen, J. G., Persson, S. E., Elias, J. H., \& Frogel, J. A. 1981, ApJ, 249, 481

Connelley, M. S., \& Greene, T. P. 2010, AJ, 140, 1214

Connelley, M. S., \& Greene, T. P. 2014, AJ, 147, 125

Connelley, M. S., Reipurth, B., \& Tokunaga, A. T. 2007, AJ, 133, 1528

Connelley, M. S., Reipurth, B., \& Tokunaga, A. T. 2008, AJ, 135, 2496 
Gregorio-Hetem, J., Lepine, J. R. D., Quast, G. R., Torres, C. A. O., \& de La Reza, R. 1992, AJ, 103, 549

Grinin, V. P., Barsunova, O. Y., Shugarov, S. Y., Kroll, P., \& Sergeev, S. G. 2008, Ap, 51, 1

Herbig, G. H. 1960, ApJS, 4, 337

Herbig, G. H. 1998, ApJ, 497, 736

Herbst, W., \& Racine, R. 1976, AJ, 81, 840

Herbst, W., \& Shevchenko, V. S. 1999, AJ, 118, 1043

Hillenbrand, L. A. 1995, PhD thesis, Univ. Massachusetts

Kim, B. G., Kawamura, A., Yonekura, Y., \& Fukui, Y. 2004, PASJ, 56, 313

Kim, M. K., Hirota, T., Honma, M., et al. 2008, PASJ, 60, 991

Kobayashi, N., Tokunaga, A. T., Terada, H., et al. 2000, SPIE 4008, 1056

Koenig, X. P., Leisawitz, D. T., Benford, D. J., et al. 2012, ApJ, 744, 130

Krisciunas, K., Sinton, W., Tholen, K., et al. 1987, PASP, 99, 887

Le Borgne, D., Rocca-Volmerange, B., Prugniel, P., et al. 2004, A\&A, 425,881

Lee, H.-T., \& Chen, W. P. 2009, ApJ, 694, 1423

Leggett, S. K., Currie, M. J., Varricatt, W. P., et al. 2006, MNRAS, 373, 781

Lombardi, M., Alves, J., \& Lada, C. J. 2011, A\&A, 535, A16

Maddalena, R. J., Morris, M., Moscowitz, J., \& Thaddeus, P. 1986, ApJ, 303,375

Magakian, T. Y. 2003, A\&A, 399, 141

Malfait, K., Bogaert, E., \& Waelkens, C. 1998, A\&A, 331, 211

Menten, K. M., Reid, M. J., Forbrich, J., \& Brunthaler, A. 2007, A\&A, 474, 515

Muench, A., Getman, K., Hillenbrand, L., \& Preibisch, T. 2008, in Handbook of Star Forming Regions, Vol. 1, ed. B. Reipurth (San Francisco, CA: ASP Monograph Publications), 483

Muzerolle, J., D’Alessio, P., Calvet, N., \& Hartmann, L. 2004, ApJ, 617, 406
Pickles, A. J. 1998, PASP, 110, 863

Pojmanski, G. 1997, AcA, 47, 467

Pojmanski, G. 2002, AcA, 52, 397

Reipurth, B., Pedrosa, A., \& Lago, M. T. V. T. 1996, A\&AS, 120, 229

Robitaille, T. P., Whitney, B. A., Indebetouw, R., \& Wood, K. 2007, ApJS, 169,328

Robitaille, T. P., Whitney, B. A., Indebetouw, R., Wood, K., \& Denzmore, P. 2006, ApJS, 167, 256

Rucinski, S. M., Zwintz, K., Hareter, M., et al. 2010, A\&A, 522, A113

Silva, D. R., \& Cornell, M. E. 1992, ApJS, 81, 865

Simons, D. A., \& Tokunaga, A. 2002, PASP, 114, 169

Skrutskie, M. F., Cutri, R. M., Stiening, R., et al. 2006, AJ, 131, 1163

Testi, L., Palla, F., \& Natta, A. 1998, A\&AS, 133, 81

Testi, L., Palla, F., \& Natta, A. 1999, A\&A, 342, 515

Thé, P. S., de Winter, D., \& Perez, M. R. 1994, A\&AS, 104, 315

Tody, D. 1986, SPIE 627, 733

Tokunaga, A. T. 2000, in Allen's Astrophysical Quantities, ed. A. Cox (New York: Springer), 143

Tokunaga, A. T., Kobayashi, N., Bell, J., et al. 1998, SPIE, 3354, 512

Tokunaga, A. T., Simons, D. A., \& Vacca, W. D. 2002, PASP, 114, 180

Uehara, M., Nagashima, C., Sugitani, K., et al. 2004, SPIE 5492, 661

van den Bergh, S. 1966, AJ, 71, 990

van Dokkum, P. G. 2001, PASP, 113, 1420

Vieira, S. L. A., Corradi, W. J. B., Alencar, S. H. P., et al. 2003, AJ, 126, 2971

Vogt, S. S., Allen, S. L., Bigelow, B. C., et al. 1994, SPIE 2198, 362

Wainscoat, R. J., \& Cowie, L. L. 1992, AJ, 103, 332

Wilson, B. A., Dame, T. M., Masheder, M. R. W., \& Thaddeus, P. 2005, A\&A, 430, 523

Wright, E. L., Eisenhardt, P. R. M., Mainzer, A. K., et al. 2010, AJ, 140, 1868 